\title{
LOCAL SYMMETRY OF HARMONIC SPACES AS DETERMINED BY THE SPECTRA OF SMALL GEODESIC SPHERES
}

\author{
TERESA ARIAS-MARCO AND DOROTHEE SCHUETH
}

\begin{abstract}
We show that in any harmonic space, the eigenvalue spectra of the Laplace operator on small geodesic spheres around a given point determine the norm $|\nabla R|$ of the covariant derivative of the Riemannian curvature tensor in that point. In particular, the spectra of small geodesic spheres in a harmonic space determine whether the space is locally symmetric. For the proof we use the first few heat invariants and consider certain coefficients in the radial power series expansions of the curvature invariants $|R|^{2}$ and $|\mathrm{Ric}|^{2}$ of the geodesic spheres. Moreover, we obtain analogous results for geodesic balls with either Dirichlet or Neumann boundary conditions. We also comment on the relevance of these results to constructions of Z.I. Szabó.
\end{abstract}

\section{INTRODUCTION}

For a compact closed Riemannian manifold $S$ the spectrum of $S$ is the eigenvalue spectrum, including multiplicities, of the associated (positive semi-definite) Laplace operator $\Delta$ acting on smooth functions. A central question of inverse spectral geometry asks to which extent the geometry of $S$ is determined by its spectrum. The so-called heat invariants $a_{k}(S)$ of $S$ are examples of geometric invariants which are determined by the spectrum of $S$; indeed, they are the coefficients in the famous asymptotic expansion by Minakshisundaram-Pleijel,

$$
\operatorname{Tr}(\exp (-t \Delta)) \sim(4 \pi t)^{-\operatorname{dim}(S) / 2} \sum_{k=0}^{\infty} a_{k}(S) t^{k}
$$

for $t \downarrow 0$. The first few of these coefficients are given by

$$
a_{0}(S)=\operatorname{vol}(S), \quad a_{1}(S)=\frac{1}{6} \int_{S} \operatorname{scal} v_{v o l}, \quad a_{2}(S)=\frac{1}{360} \int_{S}\left(5 \mathrm{scal}^{2}-\left.2\left|\mathrm{Ric}^{2}+2\right| R\right|^{2}\right) d v o l_{S},
$$

where scal, Ric, and $R$ denote the scalar curvature, the Ricci operator, and the Riemannian curvature operator of $S$, respectively. In general, each $a_{k}(S)$ is the integral over $S$ of certain curvature invariants; see [9] for more information.

Nevertheless, there exist many examples of pairs or families of isospectral Riemannian manifolds (i.e., sharing the same spectrum) which are not isometric, sometimes not even locally isometric; see, for example, the survey article [10]. Still, many questions remain open; for example, it is not

2000 Mathematics Subject Classification. 53C25, 53C20, 58J50, 58J53, 53C30, 22E25.

Key words and phrases. Harmonic space, curvature invariants, second fundamental form, Ledger's recursion formula, geodesic spheres, geodesic balls, heat invariants, isospectral manifolds, Damek-Ricci spaces.

The authors were partially supported by DFG Sonderforschungsbereich 647. The first author's work has also been supported by D.G.I. (Spain) and FEDER Project MTM2010-15444, by Junta de Extremadura and FEDER funds, and the program "Estancias de movilidad en el extranjero 'José Castillejo' para jóvenes doctores" of the Ministry of Education (Spain).

Dedication. Dorothee Schueth would like to dedicate this article to her former high school teacher, Martin Berg. It was he who first made her see the beauty of Mathematics. 
known whether a locally symmetric compact closed Riemannian manifold can be isospectral to a locally nonsymmetric Riemannian manifold.

On the other hand, the geometry of geodesic spheres plays an interesting role in Riemannian geometry. Chen and Vanhecke 4 formulated the following general question: To what extent do the properties of small geodesic spheres determine the Riemannian geometry of the ambient space? For example, Gray and Vanhecke [12] studied the information contained in the volume function of small geodesic spheres and investigated the question whether a Riemannian manifold whose geodesic spheres have the same volumes as spheres in euclidean space must necessarily be flat (answering this question in the positive under various choices of additional assumptions).

In the context of inverse spectral geometry, an interesting special version of the above question is: To what extent do the spectra of small geodesic spheres in a (possibly noncompact) Riemannian manifold $M$ determine the geometry of $M$ ? For example, Theorem 6.18 in [4] uses the information contained in the heat invariants $a_{0}$ and $a_{1}$ of small geodesic spheres (viewed as functions of the radius) and concludes local isometry of manifolds with adapted holonomy to certain model spaces under the assumption that all small geodesic spheres around each point are isospectral to the corresponding geodesic spheres in those model spaces.

In order to arrive at such and similar results, one uses radial power series expansions of curvature invariants, both of the ambient space and of the geodesic spheres. In general, even the first few coefficients of such expansions become very complicated; see, for example, the various formulas in [12] or [4]. One setting in which a quite restrictive geometric assumption on the ambient space makes the calculations considerably easier is the setting of harmonic ambient spaces.

A manifold is called harmonic if the volume density function of the geodesic exponential map is radial around each point. The notion of harmonicity was first introduced by Copson and Ruse [5] and intensively studied by Lichnerowicz [13]; see also [16]. Chapter 6 of the book by Besse [2] gives a useful survey of properties of harmonic spaces. One of the important facts about harmonic spaces is that they are Einstein [2] and hence analytic [7] (the latter result was not yet known when [2] was written). A locally symmetric manifold is harmonic if and only if it is flat or of rank one; the famous Lichnerowicz conjecture postulated that, conversely, each harmonic space is locally symmetric; i.e., satisfies $\nabla R=0$ (this condition is classically known to be equivalent to the condition that the local geodesic symmetries around each point be isometries). For the case of compact manifolds with finite fundamental group the Lichnerowicz conjecture was proved by Szabó [18; however, Damek and Ricci gave examples of noncompact homogeneous harmonic manifolds which are not locally symmetric in infinitely many dimensions greater or equal to seven [6]. These spaces are usually referred to as Damek-Ricci spaces; see [1] for more information.

Specializing the above question about the information contained in the spectra of small geodesic spheres to the setting of harmonic spaces, we are able to prove in the present paper that the spectra of small geodesic spheres in a harmonic space determine whether the space is locally symmetric (see Corollary 1.2 below). More precisely, we obtain:

Main Theorem 1.1. Let $M_{1}$ and $M_{2}$ be harmonic spaces, and let $p_{1} \in M_{1}, p_{2} \in M_{2}$. If there exists $\varepsilon>0$ such that for each $r \in(0, \varepsilon)$ the geodesic spheres $S_{r}\left(p_{1}\right)$ and $S_{r}\left(p_{2}\right)$ are isospectral, then $|\nabla R|_{p_{1}}^{2}=|\nabla R|_{p_{2}}^{2}$.

Corollary 1.2. Let $M_{1}$ and $M_{2}$ be harmonic spaces. Assume that the hypothesis of Theorem 1.1 is satisfied for each pair of points $p_{1} \in M_{1}, p_{2} \in M_{2}$. Then $M_{1}$ is locally symmetric if and only if $M_{2}$ is locally symmetric. 
In particular, note that in the case of locally homogeneous harmonic spaces $M_{1}$ and $M_{2}$, the hypothesis of Theorem 1.1 implies that $M_{1}$ is locally symmetric if and only if $M_{2}$ is locally symmetric. Actually, all known examples of harmonic spaces are locally homogeneous; it is an open question whether there exist harmonic spaces which are not locally homogeneous.

Interestingly, our result implies that certain pairs of geodesic spheres which were claimed to be isospectral by Szabó in [20], 21] are actually not isospectral. In fact, Szabó considered (as the featured examples in a more general construction) geodesic spheres in certain symmetric spaces $M_{1}$ (namely, quaternionic hyperbolic space of real dimension $4 m \geq 12$ ) and in certain associated locally nonsymmetric Damek-Ricci spaces $M_{2}$ of the same dimension (see also Remark 2.4). He stated that every pair of geodesic spheres $S_{r}\left(p_{1}\right) \subset M_{1}$ and $S_{r}\left(p_{2}\right) \subset M_{2}$ of the same radius was isospectral. Since these ambient manifolds $M_{1}$ and $M_{2}$ are harmonic and homogeneous, and $M_{1}$ is locally symmetric while $M_{2}$ is not, Corollary 1.2 immediately implies that Szabó's result cannot be correct. Note that it was Fürstenau [8] who first discovered that actually there was a gap in Szabó's isospectrality argument. The question of whether that proof could be repaired or not had since remained open; our result settles this question in the negative.

The incorrect examples of geodesic spheres mentioned above had the notable property that one is homogeneous and the other not. While it remains unknown whether a homogeneous metric on a sphere can be isospectral to a non-homogeneous one, Szabó in an earlier article [19] did construct a pair of isospectral metrics, only one of which is homogeneous, on the product of a sphere and a torus (those results are not affected by the error in the later papers).

We obtain analogs of our above results for geodesic balls endowed with either Dirichlet or Neumann boundary conditions; see Theorem 5.1 and Corollary [5.2. Similarly as above, this implies that Szabó's examples in [20, 21] of isospectral geodesic balls (of any given radius) in quaternionic hyperbolic space of real dimension at least 12 and in certain associated locally nonsymmetric Damek-Ricci spaces were erroneous.

Note that nevertheless there do exist isospectral pairs and even continuous families of isospectral metrics on spheres and balls; the first such examples were due to Gordon [11].

In order to prove Theorem 1.1 we use the heat invariants $a_{0}\left(S_{r}(p)\right)$ and $a_{2}\left(S_{r}(p)\right)$ of geodesic spheres in harmonic spaces. In particular, we study the coefficients of $r^{2}$ in the radial power series expansions of $\frac{1}{\operatorname{vol}\left(S_{r}(p)\right)} \int_{S_{r}(p)}\left|\operatorname{Ric}^{S}\right|^{2} d v l_{S_{r}(p)}$ and $\frac{1}{\operatorname{vol}\left(S_{r}(p)\right)} \int_{S_{r}(p)}\left|R^{S}\right|^{2} d v o l_{S_{r}(p)}$, where $\operatorname{Ric}^{S}$ and $R^{S}$ denote the Ricci operator and the Riemannian curvature operator of $S_{r}(p)$. From the form of these coefficients (see Proposition 3.2 and its mean value version Proposition 4.2), we are able to conlude that the heat invariants $a_{0}$ and $a_{2}$ of $S_{r}(p)$, viewed as functions of $r$, together determine the value of $|\nabla R|^{2}$ at the midpoint $p$. Note that the same is not true for $a_{0}\left(S_{r}(p)\right)$ alone; see Remark 2.4. Moreover, in the harmonic setting, the function $r \mapsto a_{1}\left(S_{r}(p)\right)=\frac{1}{6} \int_{S_{r}(p)} \operatorname{scal}^{S} d v o l_{S_{r}(p)}$ does actually not contain more information than $r \mapsto a_{0}\left(S_{r}(p)\right)=\operatorname{vol}\left(S_{r}(p)\right)$; see Remark 2.3. So it is indeed necessary for our purpose to consider $a_{2}\left(S_{r}(p)\right)$.

Our computations rely heavily on the harmonicity of the ambient space. Note that they are related to certain more general computations in [12] and [4]; for example, Theorem 8.1 of [4] actually includes a kind of analog to our Proposition 3.2, and this even for general, not only for harmonic manifolds; however, that theorem contains information only on the coefficients of $r^{j}$ with $j \leq 0$, while we need the coefficients of $r^{2}$. In fact, in the harmonic case, the lower order coefficients turn out to be determined already by the function $r \mapsto a_{0}\left(S_{r}(p)\right)=\operatorname{vol}\left(S_{r}(p)\right)$; see Proposition 3.2 and Remark 2.3(i).

This paper is organized as follows: 
In Section 2 we gather the necessary background on harmonic spaces, mostly following [2]; in particular, we recall Ledger's recursion formula for the power series expansion of the second fundamental form of geodesic spheres, and the resulting curvature identities in harmonic spaces.

In Section 3, we study the coefficients of $r^{j}$ for $j \leq 2$ in $\left|\operatorname{Ric}^{S_{r}(p)}\right|_{\exp (r u)}^{2}$ and $\left|R^{S_{r}(p)}\right|_{\exp (r u)}^{2}$ for unit tangent vectors $u$ of harmonic spaces, using the power series expansion of the second fundamental form and its radial covariant derivative, as well as the Taylor series expansion of the Riemannian curvature tensor. Proposition 3.2 is the main result of this section.

Section 4 is devoted to the proof of the Main Theorem 1.1. In preparation for this, we first derive a mean value version of Proposition 3.2, see Proposition 4.2

Finally, in Section 5, we prove the analog of Theorem 1.1 for geodesic balls. We consider the heat coefficients of geodesic balls in harmonic spaces and show that the functions $r \mapsto a_{0}\left(B_{r}(p)\right)$ and $r \mapsto a_{2}\left(B_{r}(p)\right)$ (either for Dirichlet or for Neumann boundary conditions) together determine the value of $|\nabla R|^{2}$ at the midpoint $p$ of the balls. More precisely, we show that the coefficient of $r^{3}$ in the radial power series expansion of the quotient $a_{2}\left(B_{r}(p)\right) / a_{0}\left(B_{r}(p)\right)$ is a sum of a nonzero multiple of $|\nabla R|_{p}^{2}$ and of terms determined by the function $r \mapsto a_{0}\left(B_{r}(p)\right)$.

\section{Preliminaries}

\subsection{Volume density and the shape operator of geodesic spheres.}

In the following, let $M$ be a complete, connected, $n$-dimensional Riemannian manifold. For $p \in M$, let $\exp _{p}=\left.\exp \right|_{T_{p} M}: T_{p} M \rightarrow M$ denote the associated geodesic exponential map. For a vector $v \in T_{p} M$ we denote by $\gamma_{v}$ the geodesic with initial velocity $v$. Identifying $T_{v}\left(T_{p} M\right)$ with $T_{p} M$, we regard the differential $d\left(\exp _{p}\right)_{v}$ as a linear map from $T_{p} M$ to $T_{\exp v} M$. We denote parallel translation along $\gamma_{v}$ by $P_{\gamma_{v}}^{s, t}: T_{\gamma_{v}(s)} M \rightarrow T_{\gamma_{v}(t)} M$. Given any unit vector $u \in S_{1}\left(0_{p}\right):=\{u \in$ $\left.T_{p} M|| u \mid=1\right\}$ and $r \in \mathbb{R}$, we consider the volume density

$$
\theta_{u}(r):=\operatorname{det}\left(P_{\gamma_{u}}^{r, 0} \circ d\left(\exp _{p}\right)_{r u}\right)
$$

Note that $\theta_{u}(r)$ is the infinitesimal volume distortion of the map $\exp _{p}$ at the point $r u \in T_{p} M$. Recall the Gauss lemma: The vector $d\left(\exp _{p}\right)_{r u} u$ is a unit vector perpendicular to each $d\left(\exp _{p}\right)_{r u} w$ with $w \perp u$. Thus, for each $r \in(0, i(p))$, where $i(p)$ denotes the injectivity radius of $M$ at $p$,

$$
v_{u}(r):=r^{n-1} \theta_{u}(r)
$$

is the infinitesimal volume distortion at $u$ of the map

$$
S_{1}\left(0_{p}\right) \ni u \mapsto \gamma_{u}(r)=\exp (r u) \in S_{r}(p),
$$

where $S_{r}(p) \subset M$ denotes the geodesic sphere of radius $r$ around $p$. Let $\sigma_{u}(r)$ denote the shape operator of $S_{r}(p)$ at $\exp (r u)$; that is,

$$
\sigma_{u}(r):=\left.(\nabla \nu)\right|_{T_{\exp (r u)} M},
$$

where $\nabla$ is the Levi-Civita connection of $M$ and $\nu$ denotes the outward pointing unit normal vector field on the geodesic ball $B_{i(p)}(p) \backslash\{p\}$. In particular, $\nu \circ \gamma_{u}=\dot{\gamma}_{u}, \sigma_{u} \nu=0$, and the image of $\sigma_{u}(r)$ is contained in $T_{\gamma_{u}(r)} S_{r}(p)$. It is well-known that for all $r \in(0, i(p))$,

$$
v_{u}^{\prime}(r) / v_{u}(r)=\operatorname{Tr}\left(\sigma_{u}(r)\right),
$$

and that the covariant derivative $\sigma_{u}^{\prime}$ of the endomorphism field $\sigma_{u}$ along $\left.\gamma_{u}\right|_{(0, i(p))}$ satisfies the so-called Riccati equation

$$
\sigma_{u}^{\prime}=-\sigma_{u}^{2}-R_{\dot{\gamma}_{u}}
$$


where $R$ is the Riemannian curvature tensor of $M$, given by $R(x, y) z=-\nabla_{x} \nabla_{y} z+\nabla_{y} \nabla_{x} z+\nabla_{[x, y]} z$, and where $R_{\nu}:=R(\nu,.) \nu$. (Note that here we use the same sign for $R$ as Besse [2].) Let $C_{u}(r):=r \sigma_{u}(r)$. This endomorphism field along $\left.\gamma_{u}\right|_{(0, i(p))}$ is smoothly extendable to $r=0$ by $C_{u}(0):=I_{u}$, where $I_{u}$ is defined by $I_{u}(u)=0$ and $\left.I_{u}\right|_{\{u\}^{\perp}}=\operatorname{Id}_{\{u\}^{\perp}}$. Moreover, from (3) one can derive Ledger's recursion formula for the covariant derivatives of $C_{u}$ at $r=0$ (see, e.g., [4]):

$$
(k-1) C_{u}^{(k)}(0)=-k(k-1) R_{u}^{(k-2)}-\sum_{\ell=0}^{k}\left(\begin{array}{l}
k \\
\ell
\end{array}\right) C_{u}^{(\ell)}(0) C_{u}^{(k-\ell)}(0)
$$

for all $k \in \mathbb{N}$, where $R_{u}^{(k)}$ is the $k$-th covariant derivative of the endomorphism field $R_{\dot{\gamma}_{u}}$ along $\gamma_{u}$ at $r=0$. This formula allows one to successively compute the $C_{u}^{(k)}(0)$ in terms of the endomorphisms $R_{u}^{(k)}$ of $T_{p} M$. Forming the Taylor series of $C_{u}$ and dividing by $r$, one obtains (see, e.g., [2], [4]):

$$
\begin{aligned}
P_{\gamma_{u}}^{r, 0} \circ \sigma_{u}(r) \circ P_{\gamma_{u}}^{0, r}= & \frac{1}{r} I_{u}-\frac{r}{3} R_{u}-\frac{r^{2}}{4} R_{u}^{\prime}-\left(\frac{1}{10} R_{u}^{\prime \prime}+\frac{1}{45} R_{u} R_{u}\right) r^{3} \\
& -\left(\frac{1}{36} R_{u}^{\prime \prime \prime}+\frac{1}{72} R_{u} R_{u}^{\prime}+\frac{1}{72} R_{u}^{\prime} R_{u}\right) r^{4} \\
& -\left(\frac{1}{168} R_{u}^{(4)}+\frac{1}{210} R_{u} R_{u}^{\prime \prime}+\frac{1}{210} R_{u}^{\prime \prime} R_{u}+\frac{1}{112} R_{u}^{\prime} R_{u}^{\prime}+\frac{2}{945} R_{u} R_{u} R_{u}\right) r^{5} \\
& +O\left(r^{6}\right) .
\end{aligned}
$$

\subsection{Curvature identities in harmonic spaces.}

The manifold $M$ is called a harmonic space if for every $p \in M$ the above function $\theta_{u}$ does not depend on $u \in S_{1}\left(0_{p}\right)$. An equivalent condition is that for all $r \in(0, i(p))$, the geodesic spheres $S_{r}(p)$ have constant mean curvature (recall equations (1), (2) ). For more information on harmonic spaces see [16] or [2]. If $M$ is harmonic then the function $\theta_{u}$ does in fact not even depend on $p$; that is, there exists $\theta:[0, \infty) \rightarrow \mathbb{R}$ such that

$$
\theta_{u}(r)=\theta(r)
$$

for all $u \in T M$ with $|u|=1$. Moreover, even the local or infinitesimal versions of the above condition imply that the manifold is Einstein [2] and therefore analytic [7]. Hence, the local or infinitesimal versions of the above conditions are equivalent to the global versions. Since $\theta_{u}(r)$ depends only on $r$, so does $v_{u}(r)$ and hence $\operatorname{Tr}\left(\sigma_{u}(r)\right)$. From this one can successively derive, using the expansion (4):

Proposition 2.1 (see [2], Chapter 6). If $M$ is harmonic then there exist constants $C, H, L \in \mathbb{R}$ such that for all $p \in M$ and all $u \in T_{p} M$ with $|u|=1$ :

(i) $\operatorname{Tr}\left(R_{u}\right)=C$; in particular:

(ii) $\operatorname{Tr}\left(R_{u}^{(k)}\right)=0$ for all $k \in \mathbb{N}$.

(iii) $\operatorname{Tr}\left(R_{u} R_{u}\right)=H$; in particular:

(iv) $\operatorname{Tr}\left(R_{u} R_{u}^{\prime}\right)=0$ and

(v) $\operatorname{Tr}\left(R_{u} R_{u}^{\prime \prime}\right)=-\operatorname{Tr}\left(R_{u}^{\prime} R_{u}^{\prime}\right)$.

(vi) $\operatorname{Tr}\left(32 R_{u} R_{u} R_{u}-9 R_{u}^{\prime} R_{u}^{\prime}\right)=L$.

In fact, taking traces in (4), one has in the harmonic case:

$$
\operatorname{Tr}\left(\sigma_{u}(r)\right)=(n-1) \frac{1}{r}-\frac{1}{3} C r-\frac{1}{45} H r^{3}-\frac{1}{15120} L r^{5}+O\left(r^{7}\right)
$$


for $r \downarrow 0$ and all $u \in T M$ with $|u|=1$. Note that Proposition 2.1(i) just says that the Einstein constant of $M$ is $C$; that is, Ric $=C$ Id on each $T_{p} M$. Recall that the Ricci operator is defined by $\langle\operatorname{Ric}(x), y\rangle=\operatorname{Tr}(R(x,) y$.$) for all x, y \in T_{p} M$ and all $p \in M$. From Proposition 2.1 one can further derive:

Proposition 2.2 (see [2], Chapter 6). If $M$ is harmonic, then for the above constants $C, H, L$ and each $p \in M$ :

(i) $\langle R(x,.) ., R(y,)\rangle=.\frac{2}{3}\left((n+2) H-C^{2}\right)\langle x, y\rangle$ for all $x, y \in T_{p} M$; in particular:

(ii) $|R|_{p}^{2}=\frac{2}{3} n\left((n+2) H-C^{2}\right)$.

(iii) $32\left(n C^{3}+\frac{9}{2} C|R|_{p}^{2}+\frac{7}{2} \hat{R}(p)-\stackrel{\circ}{R}(p)\right)-27|\nabla R|_{p}^{2}=n(n+2)(n+4) L$.

Here, the functions $\hat{R}, \stackrel{\circ}{R} \in C^{\infty}(M)$ are certain curvature invariants of order six which are defined as follows: If $\left\{e_{1}, \ldots, e_{n}\right\}$ is an orthonormal basis of $T_{p} M$ and $R_{i j k \ell}:=\left\langle R\left(e_{i}, e_{j}\right) e_{k}, e_{\ell}\right\rangle$, then

$$
\stackrel{\circ}{R}(p):=\sum_{i, j, k, \ell, a, b} R_{i j k \ell} R_{j a \ell b} R_{a i b k}, \quad \hat{R}(p):=\sum_{i, j, k, \ell, a, b} R_{i j k \ell} R_{k \ell a b} R_{a b i j} .
$$

Note that the term $n C^{3}$ in Proposition 2.2 (iii) reads $n C^{2}$ in the corresponding equation 6.67 in [2], but this was obviously a misprint (note that curvature terms of different order cannot occur here); see also formula (3.1) in [22].

Proposition 2.2(iii) will be used in Section 4, together with the following formula which actually holds in any Einstein manifold; see formula (6-7) in [14] or formula (11.3) in [12]:

$$
-\frac{1}{2} \Delta\left(|R|^{2}\right)=2 C|R|^{2}-\hat{R}-4 \stackrel{\circ}{R}+|\nabla R|^{2},
$$

where $\Delta$ denotes the Laplace operator on functions, that is, $\Delta f=-\sum_{i}\left(e_{i}\left(e_{i} f\right)-\left(\nabla_{e_{i}} e_{i}\right) f\right)$ for local orthonormal frames $\left\{e_{1}, \ldots, e_{n}\right\}$. (Again, there is a misprint in two of the coefficients in the corresponding formula 6.65 in [2].) If $M$ is harmonic, then the left hand side of (6) is zero by Proposition 2.2(ii). Finally, we recall the following well-known observations which will be used in Section 4

Remark 2.3. Let $M$ be an $n$-dimensional harmonic space with volume density function $\theta$ as above.

(i) For any $p \in M$, the volume of the geodesic sphere $S_{r}(p)$ with $0<r<i(p)$ equals the volume $\omega_{n-1}$ of the standard unit sphere $S^{n-1}$ in $\mathbb{R}^{n}$ multiplied by the factor

$$
v(r):=r^{n-1} \theta(r)
$$

Note that $v(r)=v_{u}(r)$ for each unit vector $u \in T M$, where $v_{u}$ is the function defined in (1). The function $v$ determines the volume growth function $v^{\prime} / v$ of the geodesic spheres, and thus it determines, by (2), the function $\operatorname{Tr}\left(\sigma_{u}(r)\right.$ ) (which is independent of $u$ ). By (5), the function which associates to small values of $r$ the volume of geodesic spheres of radius $r$ in a given harmonic space $M$ determines the constants $C, H, L$ (and of course $n$ ) associated with $M$.

(ii) Let scal $=n C$ denote the scalar curvature of $M$. Let $p \in M$, fix some $r \in(0, i(p))$, and let $\mathrm{scal}^{S}$ denote the scalar curvature function of $S_{r}(p)$. A routine calculation using the Gauss equation shows that for each unit vector $u \in T_{p} M$ we have

$$
\operatorname{scal}^{S}(\exp (r u))=\operatorname{scal}-2\left\langle\operatorname{Ric}\left(\dot{\gamma}_{u}(r)\right), \dot{\gamma}_{u}(r)\right\rangle+\left(\operatorname{Tr}\left(\sigma_{u}(r)\right)\right)^{2}-\operatorname{Tr}\left(\sigma_{u}(r)^{2}\right)
$$


which by the Einstein condition and equations (2) and (3) implies

$$
\begin{aligned}
\operatorname{scal}^{S}(\exp (r u)) & =(n-2) C+\left(v^{\prime}(r) / v(r)\right)^{2}+\operatorname{Tr}\left(\sigma_{u}^{\prime}(r)\right)+\operatorname{Tr}\left(R_{\dot{\gamma}_{u}(r)}\right) \\
& =(n-2) C+\left(v^{\prime}(r) / v(r)\right)^{2}+\left(v^{\prime} / v\right)^{\prime}(r)+C=(n-1) C+v^{\prime \prime}(r) / v(r) .
\end{aligned}
$$

Therefore, geodesic spheres in $M$ have constant scalar curvature, and the respective constant depends only on the radius, not on the midpoint. Finally, using (i) one concludes that the function which associates to small values of $r$ the scalar curvature of geodesic spheres of radius $r$ is determined already by the function which associates to small values of $r$ the volume of geodesic spheres of radius $r$.

Remark 2.4. As mentioned in the Introduction, the aim of this paper is to show that in harmonic spaces, the heat invariants $a_{0}\left(S_{r}(p)\right)=\operatorname{vol}\left(S_{r}(p)\right)$ and $a_{2}\left(S_{r}(p)\right)$, viewed as functions of $r$, together determine $|\nabla R|_{p}^{2}$. This is not the case for $a_{0}$ alone, as manifested by certain pairs of Damek-Ricci spaces. A Damek-Ricci space $A N$ is a certain type of solvable Lie groups with left invariant metric, namely, the standard 1-dimensional solvable extension of a simply connected Riemannian nilmanifold $N$ of Heisenberg type. The volume density function of $A N$ is radial and depends only on the dimensions of $N$ and its center [6]; see also the book [1]. Within the class of DamekRicci spaces, there exist pairs of symmetric spaces $A N$ and locally nonsymmetric spaces $A N^{\prime}$ where $N$ and $N^{\prime}$ have the same dimension and so do their centers. (In fact, certain such pairs $A N$ and $A N^{\prime}$ were the ambient manifolds used by Szabó in [20], 21]; recall the Introduction.) In particular, geodesic spheres of the same radius in $A N$ and $A N^{\prime}$ have the same volume. This shows that in harmonic spaces, the function $r \mapsto \operatorname{vol}\left(S_{r}(p)\right)$ alone does not determine $|\nabla R|_{p}^{2}$. In turn, Remark 2.3 shows that in harmonic spaces, the function $r \mapsto a_{0}\left(S_{r}(p)\right)=\operatorname{vol}\left(S_{r}(p)\right)$ already determines the function $r \mapsto a_{1}\left(S_{r}(p)\right)=\frac{1}{6} \int_{S_{r}(p)} \operatorname{scal}^{S} d v o l_{S_{r}(p)}$. Therefore, we need to consider $a_{2}\left(S_{r}(p)\right)$. The next section gives some necessary preparations for this.

\section{RAdial EXPANSIONS OF $\mid$ Ric $\left.\right|^{2}$ AND $|R|^{2}$ FOR GEODESIC SPHERES IN HARMONIC SPACES}

In this section we will describe a certain coefficient in the radial power series expansions of the curvature invariants $\mid$ Ric $\left.\right|^{2}$ and $|R|^{2}$ of geodesic spheres in harmonic spaces. First we need the following lemma.

Lemma 3.1. Let $M$ be an $n$-dimensional harmonic space, and let $C$ and $H$ be the constants from Proposition 2.1. Let $p \in M$, and let $S:=S_{r}(p)$ be a geodesic sphere around $p$ with radius $r \in(0, i(p))$, endowed with the induced Riemannian metric. Let $u$ be a unit vector in $T_{p} M$, let $\sigma:=\sigma_{u}(r)$ be as in Section 2, and write $\sigma^{\prime}:=\sigma_{u}^{\prime}(r)$. Let $R^{S}$ and $\mathrm{Ric}^{S}$ denote the curvature tensor, resp. the Ricci operator, of $S$. Then in the point $q:=\exp (r u) \in S$ we have:

(i) $\left|\operatorname{Ric}^{S}\right|_{q}^{2}=(n-1) C^{2}+2 C(\operatorname{Tr}(\sigma))^{2}+(\operatorname{Tr}(\sigma))^{2} \operatorname{Tr}\left(\sigma^{2}\right)+2 C \operatorname{Tr}\left(\sigma^{\prime}\right)+2 \operatorname{Tr}(\sigma) \operatorname{Tr}\left(\sigma \sigma^{\prime}\right)+\operatorname{Tr}\left(\sigma^{\prime} \sigma^{\prime}\right)$,

(ii) $\left|R^{S}\right|_{q}^{2}=\frac{2}{3}(n-4)\left((n+2) H-C^{2}\right)+4 H+2\left(\operatorname{Tr}\left(\sigma^{2}\right)\right)^{2}-2 \operatorname{Tr}\left(\sigma^{4}\right)+4 \sum_{i=1}^{n} \operatorname{Tr}\left(\sigma \circ R\left(e_{i},.\right) \sigma e_{i}\right)$,

where $\left\{e_{1}, \ldots, e_{n}\right\}$ is an orthonormal basis of $T_{q} M$.

Proof. (i) Let $\nu$ be the outward pointing radial unit vector field as in Section 2, From the Gauss equation one easily derives the following formula whose analog is valid for submanifolds of codimension one in arbitrary Riemannian manifolds:

$$
\operatorname{Ric}_{q}^{S}=\left.\left(\operatorname{Ric}-R_{\nu_{q}}+\operatorname{Tr}(\sigma) \sigma-\sigma^{2}\right)\right|_{T_{q} S}
$$


Using the Einstein condition and the Riccati equation (3), this formula becomes in our situation:

$$
\operatorname{Ric}_{q}^{S}=\left.\left(C \operatorname{Id}+\operatorname{Tr}(\sigma) \sigma+\sigma^{\prime}\right)\right|_{T_{q} S}
$$

(see also [15], p. 67). Now one obtains the desired formula immediately, keeping in mind that both $\sigma$ and $\sigma^{\prime}$ are symmetric and annihilate $\nu_{q}$.

(ii) Choose an orthonormal basis $\left\{e_{1}, \ldots, e_{n}\right\}$ of $T_{q} M$ such that $e_{1}=\nu_{q}$. For all $i, j, k, \ell \in$ $\{2, \ldots, n\}$ we have by the Gauss equation (recall our sign convention for $R$ ):

$$
\left\langle R^{S}\left(e_{i}, e_{j}\right) e_{k}, e_{\ell}\right\rangle=\left\langle R\left(e_{i}, e_{j}\right) e_{k}, e_{\ell}\right\rangle+\left\langle\sigma e_{i}, e_{k}\right\rangle\left\langle\sigma e_{j}, e_{\ell}\right\rangle-\left\langle\sigma e_{j}, e_{k}\right\rangle\left\langle\sigma e_{i}, e_{\ell}\right\rangle .
$$

Squaring both sides and forming the sum over $i, j, k, \ell$, while recalling that $\sigma$ is symmetric and annihilates $e_{1}$, we get

$$
\begin{aligned}
\left|R^{S}\right|_{q}^{2}= & \sum_{i, j, k, \ell=2}^{n}\left\langle R\left(e_{i}, e_{j}\right) e_{k}, e_{\ell}\right\rangle^{2}+|\sigma|^{2}|\sigma|^{2}+|\sigma|^{2}|\sigma|^{2} \\
& -2\left|\sigma^{2}\right|^{2}+2 \sum_{i, j=1}^{n}\left\langle R\left(e_{i}, e_{j}\right) \sigma e_{i}, \sigma e_{j}\right\rangle-2 \sum_{i, j=1}^{n}\left\langle R\left(e_{i}, e_{j}\right) \sigma e_{j}, \sigma e_{i}\right\rangle \\
= & \sum_{i, j, k, \ell=2}^{n}\left\langle R\left(e_{i}, e_{j}\right) e_{k}, e_{\ell}\right\rangle^{2}+2\left(\operatorname{Tr}\left(\sigma^{2}\right)\right)^{2}-2 \operatorname{Tr}\left(\sigma^{4}\right)+4 \sum_{i=1}^{n} \operatorname{Tr}\left(\sigma \circ R\left(e_{i}, .\right) \sigma e_{i}\right) .
\end{aligned}
$$

The desired formula now follows from the fact that the first sum on the right hand side is equal to $|R|_{q}^{2}-4\left|R\left(e_{1}, .\right) .\right|^{2}+4\left|R_{e_{1}}\right|^{2}$ which by Proposition 2.2(i), (ii) and Proposition 2.1(iii) becomes $\frac{2}{3}(n-4)\left((n+2) H-C^{2}\right)+4 H$.

Using the radial power series expansion of $\sigma$ together with the previous lemma, we will make conclusions concerning the first few coefficients of the radial expansions of $\left|\operatorname{Ric}^{S}\right|^{2}$ and $\left|R^{S}\right|^{2}$. The following proposition will be the key of the proof of the Main Theorem 1.1. Actually we will use only the statements about $\alpha_{2}$ and $\beta_{2}$ in this proposition.

Proposition 3.2. Let $M$ be an n-dimensional harmonic space, and let $C, H$, and $L$ be the constants from Proposition 2.1. Let $p \in M$, and let $u$ be a unit vector in $T_{p} M$. Then

$$
\begin{gathered}
\left|\operatorname{Ric}^{S_{r}(p)}\right|_{\exp (r u)}^{2}=\alpha_{-4} r^{-4}+\alpha_{-2} r^{-2}+\alpha_{0}+\alpha_{2}(u) r^{2}+O\left(r^{3}\right) \text { and } \\
\left|R^{S_{r}(p)}\right|_{\exp (r u)}^{2}=\beta_{-4} r^{-4}+\beta_{-2} r^{-2}+\beta_{0}+\beta_{2}(u) r^{2}+O\left(r^{3}\right)
\end{gathered}
$$

for $r \downarrow 0$, where the coefficients $\alpha_{i}$ and $\beta_{i}$ for $i \in\{-4,-2,0\}$ are constants depending only on $n$, $C$, and $H$. Moreover,

$$
\begin{aligned}
& \alpha_{2}(u)=\hat{\alpha}_{2}+\frac{1}{16} \operatorname{Tr}\left(R_{u}^{\prime} R_{u}^{\prime}\right) \text { and } \\
& \beta_{2}(u)=\hat{\beta}_{2}+\frac{4}{9} \sum_{i=1}^{n} \operatorname{Tr}\left(R_{u} \circ R\left(e_{i}, .\right) R_{u} e_{i}\right),
\end{aligned}
$$

where $\hat{\alpha}_{2}$ and $\hat{\beta}_{2}$ are constants depending only on $n, C, H$, and $L$, and where $\left\{e_{1}, \ldots, e_{n}\right\}$ is an orthonormal basis of $T_{p} M$.

Proof. We use Lemma 3.1 together with the power series expansions (4), (5) of $\sigma:=\sigma_{u}(r)$ and $\operatorname{Tr}(\sigma)$. Let us first consider $\left|\operatorname{Ric}^{S_{r}(p)}\right|_{\exp (r u)}^{2}$ and the individual contributions of the nonconstant 

terms in Lemma 3.1(i) to its expansion. By (5) we have

$$
(\operatorname{Tr}(\sigma))^{2}=\left((n-1) \frac{1}{r}-\frac{1}{3} C r-\frac{1}{45} H r^{3}-\frac{1}{15120} L r^{5}\right)^{2}+O\left(r^{6}\right)
$$

for $r \downarrow 0$. Moreover, from the expansion (4) and Proposition 2.1 one gets

$$
\operatorname{Tr}\left(\sigma^{2}\right)=(n-1) \frac{1}{r^{2}}-\frac{2}{3} C+\frac{1}{15} H r^{2}+\frac{1}{3024} L r^{4}+O\left(r^{5}\right) .
$$

Further,

by (5), and

$$
\operatorname{Tr}\left(\sigma^{\prime}\right)=\frac{d}{d r} \operatorname{Tr}(\sigma)=-(n-1) \frac{1}{r^{2}}-\frac{1}{3} C-\frac{1}{15} H r^{2}+O\left(r^{4}\right)
$$

$$
2 \operatorname{Tr}\left(\sigma \sigma^{\prime}\right)=\frac{d}{d r} \operatorname{Tr}\left(\sigma^{2}\right)=-2(n-1) \frac{1}{r^{3}}+\frac{2}{15} H r+\frac{1}{756} L r^{3}+O\left(r^{4}\right)
$$

by (7). Using these expansions and (5), one easily checks that each of the the four individual terms $2 C(\operatorname{Tr}(\sigma))^{2},(\operatorname{Tr}(\sigma))^{2} \operatorname{Tr}\left(\sigma^{2}\right), 2 C \operatorname{Tr}\left(\sigma^{\prime}\right)$, and $2 \operatorname{Tr}(\sigma) \operatorname{Tr}\left(\sigma \sigma^{\prime}\right)$ appearing on the right hand side of Lemma 3.1 (i) has the property that the corresponding coefficients of $r^{-4}, r^{-2}, r^{0}$ depend only on $n, C, H$, the coefficient of $r^{2}$ depends only on $n, C, H, L$, and the coefficients of $r^{-3}, r^{-1}, r$ vanish.

It remains to consider the term $\operatorname{Tr}\left(\sigma^{\prime} \sigma^{\prime}\right)$ in Lemma 3.1(i). From (4) we get

$$
\begin{aligned}
P_{\gamma_{u}}^{r, 0} \circ \sigma^{\prime} \circ P_{\gamma_{u}}^{0, r}= & -\frac{1}{r^{2}} I_{u}-\frac{1}{3} R_{u}-\frac{r}{2} R_{u}^{\prime}-\left(\frac{3}{10} R_{u}^{\prime \prime}+\frac{1}{15} R_{u} R_{u}\right) r^{2} \\
& -\left(\frac{1}{9} R_{u}^{\prime \prime \prime}+\frac{1}{18} R_{u} R_{u}^{\prime}+\frac{1}{18} R_{u}^{\prime} R_{u}\right) r^{3} \\
& -\left(\frac{5}{168} R_{u}^{(4)}+\frac{1}{42} R_{u} R_{u}^{\prime \prime}+\frac{1}{42} R_{u}^{\prime \prime} R_{u}+\frac{5}{112} R_{u}^{\prime} R_{u}^{\prime}+\frac{2}{189} R_{u} R_{u} R_{u}\right) r^{4} \\
& +O\left(r^{5}\right)
\end{aligned}
$$

and thereby, using Proposition 2.1;

$$
\begin{aligned}
\operatorname{Tr}\left(\sigma^{\prime} \sigma^{\prime}\right)= & (n-1) \frac{1}{r^{4}}+\frac{2}{3} \frac{C}{r^{2}}+\frac{11}{45} H \\
& +\left(\left(-\frac{2}{21}+\frac{5}{56}-\frac{1}{5}+\frac{1}{4}\right) \operatorname{Tr}\left(R_{u}^{\prime} R_{u}^{\prime}\right)+\left(\frac{4}{189}+\frac{2}{45}\right) \operatorname{Tr}\left(R_{u} R_{u} R_{u}\right)\right) r^{2}+O\left(r^{3}\right) .
\end{aligned}
$$

The coefficient of $r^{2}$ in the latter expansion is

$$
\frac{37}{840} \operatorname{Tr}\left(R_{u}^{\prime} R_{u}^{\prime}\right)+\frac{62}{945} \operatorname{Tr}\left(R_{u} R_{u} R_{u}\right)
$$

which by Proposition 2.1(vi) turns out to be

$$
\frac{62}{32 \cdot 945} L+\left(\frac{37}{840}+\frac{9 \cdot 62}{32 \cdot 945}\right) \operatorname{Tr}\left(R_{u}^{\prime} R_{u}^{\prime}\right)=\frac{31}{15120} L+\frac{1}{16} \operatorname{Tr}\left(R_{u}^{\prime} R_{u}^{\prime}\right) .
$$

This concludes the proof of the statements concerning the expansion of $\left|\operatorname{Ric}^{S_{r}(p)}\right|_{\exp (r u)}^{2}$.

We now turn to $\left|R^{S_{r}(p)}\right|_{\exp (r u)}^{2}$ and study the individual contributions of the nonconstant terms in Lemma 3.1(ii) to its expansion. Squaring (7), we see that in the expansion of the term $2\left(\operatorname{Tr}\left(\sigma^{2}\right)\right)^{2}$ the coefficients of $r^{-4}, r^{-2}, r^{0}$ depend only on $n, C, H$, the coefficient of $r^{2}$ depends only on $n, C, H, L$, and the coefficients of $r^{-3}, r^{-1}, r$ vanish. 
Regarding the term $-2 \operatorname{Tr}\left(\sigma^{4}\right)$ we obtain from (4):

$$
\begin{array}{r}
P_{\gamma_{u}}^{r, 0} \circ \sigma^{4} \circ P_{\gamma u}^{0, r}=\frac{1}{r^{4}} I_{u}-\frac{4}{3 r^{2}} R_{u}-\frac{1}{r} R_{u}^{\prime}+\left(-\frac{2}{5} R_{u}^{\prime \prime}+\frac{26}{45} R_{u}^{2}\right)+\left(-\frac{1}{9} R_{u}^{\prime \prime \prime}+\frac{4}{9}\left(R_{u} R_{u}^{\prime}+R_{u}^{\prime} R_{u}\right)\right) r \\
+\left(-\frac{1}{42} R_{u}^{(4)}+\left(-\frac{2}{105}+\frac{1}{5}\right)\left(R_{u}^{\prime \prime} R_{u}+R_{u} R_{u}^{\prime \prime}\right)+\left(-\frac{1}{28}+\frac{3}{8}\right) R_{u}^{\prime} R_{u}^{\prime}+\left(-\frac{8}{945}+\frac{4}{45}-\frac{4}{27}\right) R_{u}^{3}\right) r^{2} \\
+O\left(r^{3}\right)
\end{array}
$$

for $r \downarrow 0$. Using Proposition 2.1 we get

$$
\begin{aligned}
-2 \operatorname{Tr}\left(\sigma^{4}\right)= & -2(n-1) \frac{1}{r^{4}}+\frac{8}{3} \frac{C}{r^{2}}-\frac{52}{45} H \\
& +\left(\left(-\frac{8}{105}+\frac{4}{5}+\frac{1}{14}-\frac{3}{4}\right) \operatorname{Tr}\left(R_{u}^{\prime} R_{u}^{\prime}\right)+\left(\frac{16}{945}-\frac{8}{45}+\frac{8}{27}\right) \operatorname{Tr}\left(R_{u} R_{u} R_{u}\right)\right) r^{2}+O\left(r^{3}\right) .
\end{aligned}
$$

The coefficient of $r^{2}$ in the latter expansion is

$$
\frac{19}{420} \operatorname{Tr}\left(R_{u}^{\prime} R_{u}^{\prime}\right)+\frac{128}{945} \operatorname{Tr}\left(R_{u} R_{u} R_{u}\right)
$$

which by Proposition 2.1(vi) equals

$$
\frac{128}{32 \cdot 945} L+\left(\frac{19}{420}+\frac{9 \cdot 128}{32 \cdot 945}\right) \operatorname{Tr}\left(R_{u}^{\prime} R_{u}^{\prime}\right)=\frac{4}{945} L+\frac{1}{12} \operatorname{Tr}\left(R_{u}^{\prime} R_{u}^{\prime}\right) .
$$

It remains to consider the term $4 \sum_{i=1}^{n} \operatorname{Tr}\left(\sigma \circ R\left(e_{i},.\right) \sigma e_{i}\right)$ in Lemma 3.1(ii). We make some preliminary observations. For $k \in \mathbb{N}_{0}$, let $R^{(k)}$, resp. Ric ${ }^{(k)}$ denote the $k$-th covariant derivative of the curvature tensor, resp. the Ricci operator, along $\gamma_{u}$ at $r=0$. We will use the the Taylor series expansion of the Riemannian curvature tensor along $\gamma_{u}$ (recall that $M$ is analytic):

$$
P_{\gamma_{u}}^{r, 0} \circ R_{\gamma_{u}(r)} \circ P_{\gamma_{u}}^{0, r}=\sum_{k=0}^{\infty} \frac{r^{k}}{k !} R^{(k)}
$$

Moreover, $\operatorname{Ric}^{(k)}=0$ for $k \geq 1$ since $M$ is Einstein. Note that $\left.\operatorname{Ric}\right|_{T_{p} M}=\sum_{i=1}^{n} R_{e_{i}}$ and similarly on each $T_{\gamma_{u}(r)} M$ if we extend $\left\{e_{1}, \ldots, e_{n}\right\}$ parallelly along $\gamma_{u}$. For any $k \in \mathbb{N}_{0}$ we have, using Proposition 2.1.

$$
\begin{aligned}
\sum_{i=1}^{n} \operatorname{Tr}\left(I_{u} \circ R^{(k)}\left(e_{i}, .\right) I_{u} e_{i}\right) & =\operatorname{Tr}\left(I_{u} \circ \operatorname{Ric}^{(k)}\right)-\operatorname{Tr}\left(I_{u} \circ R_{u}^{(k)}\right) \\
& =\operatorname{Tr}\left(\operatorname{Ric}^{(k)}\right)-\left\langle\operatorname{Ric}^{(k)} u, u\right\rangle-\operatorname{Tr}\left(R_{u}^{(k)}\right)= \begin{cases}(n-2) C, & k=0, \\
0, & k \geq 1 .\end{cases}
\end{aligned}
$$

Moreover,

$$
\begin{aligned}
\sum_{i=1}^{n} \operatorname{Tr}\left(R_{u} \circ R^{(k)}\left(e_{i}, .\right) I_{u} e_{i}\right) & =\operatorname{Tr}\left(R_{u} \circ \operatorname{Ric}^{(k)}\right)-\operatorname{Tr}\left(R_{u} R_{u}^{(k)}\right) \\
& = \begin{cases}C^{2}-H, & k=0, \\
-\operatorname{Tr}\left(R_{u} R_{u}^{(k)}\right), & k \geq 1,\end{cases}
\end{aligned}
$$




$$
\begin{gathered}
\sum_{i=1}^{n} \operatorname{Tr}\left(R_{u}^{\prime} \circ R^{(k)}\left(e_{i}, .\right) I_{u} e_{i}\right)=\operatorname{Tr}\left(R_{u}^{\prime} \circ \operatorname{Ric}^{(k)}\right)-\operatorname{Tr}\left(R_{u}^{\prime} R_{u}^{(k)}\right) \\
= \begin{cases}0, & k=0, \\
-\operatorname{Tr}\left(R_{u}^{\prime} R_{u}^{(k)}\right), & k \geq 1,\end{cases} \\
\sum_{i=1}^{n} \operatorname{Tr}\left(R_{u}^{\prime \prime} \circ R\left(e_{i}, .\right) I_{u} e_{i}\right)=\operatorname{Tr}\left(R_{u}^{\prime \prime} \circ \operatorname{Ric}\right)-\operatorname{Tr}\left(R_{u}^{\prime \prime} R_{u}\right)=0+\operatorname{Tr}\left(R_{u}^{\prime} R_{u}^{\prime}\right), \\
\sum_{i=1}^{n} \operatorname{Tr}\left(R_{u} R_{u} \circ R\left(e_{i}, .\right) I_{u} e_{i}\right)=\operatorname{Tr}\left(R_{u} R_{u} \circ \operatorname{Ric}\right)-\operatorname{Tr}\left(R_{u} R_{u} R_{u}\right)=C H-\operatorname{Tr}\left(R_{u} R_{u} R_{u}\right) .
\end{gathered}
$$

Note that for any pair of symmetric endomorphisms $F, G$ of $T_{p} M$ we have

$$
\sum_{i=1}^{n} \operatorname{Tr}\left(F \circ R^{(k)}\left(e_{i}, .\right) G e_{i}\right)=\sum_{i=1}^{n} \operatorname{Tr}\left(G \circ R^{(k)}\left(e_{i}, .\right) F e_{i}\right)
$$

by the symmetries of the curvature operator. Keeping the expansions (4) and (9) in mind, we see that the expression in (10) contributes only to the coefficient of $r^{-2}$ in the expansion of $\sum_{i=1}^{n} \operatorname{Tr}(\sigma \circ$ $\left.R\left(e_{i},.\right) \sigma e_{i}\right)$, the expression in (11) contributes to the coefficients of $r^{0}$ and $r^{2}$ (and higher order), the expressions in (12), (13), (14) contribute to the coefficient of $r^{2}$ (and higher order). The only additional contribution to the coefficient of $r^{2}$ is given by the sum of $\operatorname{Tr}\left(R_{u} \circ R\left(e_{i},.\right) R_{u} e_{i}\right)$. Recalling (15) (and multiplying $R^{(k)}$ by $1 / k$ !), we obtain from (4), 2.1(iv), (9), and the above observations:

$$
\begin{aligned}
4 \sum_{i=1}^{n} \operatorname{Tr}\left(\sigma \circ R\left(e_{i}, .\right) \sigma e_{i}\right)= & 4\left((n-2) \frac{C}{r^{2}}-\frac{2}{3}\left(C^{2}-H\right)\right. \\
+ & {\left[\frac{2}{3 \cdot 2 !} \operatorname{Tr}\left(R_{u} R_{u}^{\prime \prime}\right)+\left(\frac{2}{4}-\frac{2}{10}\right) \operatorname{Tr}\left(R_{u}^{\prime} R_{u}^{\prime}\right)-\frac{2}{45} C H+\frac{2}{45} \operatorname{Tr}\left(R_{u} R_{u} R_{u}\right)\right.} \\
& \left.\left.+\frac{1}{9} \sum_{i=1}^{n} \operatorname{Tr}\left(R_{u} \circ R\left(e_{i}, .\right) R_{u} e_{i}\right)\right] r^{2}\right)+O\left(r^{3}\right) .
\end{aligned}
$$

By Proposition 2.1(v), the coefficient of $r^{2}$ in the latter expansion is

$$
-\frac{8}{45} C H-\frac{2}{15} \operatorname{Tr}\left(R_{u}^{\prime} R_{u}^{\prime}\right)+\frac{8}{45} \operatorname{Tr}\left(R_{u} R_{u} R_{u}\right)+\frac{4}{9} \sum_{i=1}^{n} \operatorname{Tr}\left(R_{u} \circ R\left(e_{i}, .\right) R_{u} e_{i}\right)
$$

By Proposition 2.1(vi), the two terms involving $\operatorname{Tr}\left(R_{u}^{\prime} R_{u}^{\prime}\right)$ and $\operatorname{Tr}\left(R_{u} R_{u} R_{u}\right)$ become

$$
\frac{8}{32 \cdot 45} L+\left(-\frac{2}{15}+\frac{9 \cdot 8}{32 \cdot 45}\right) \operatorname{Tr}\left(R_{u}^{\prime} R_{u}^{\prime}\right)=\frac{1}{180} L-\frac{1}{12} \operatorname{Tr}\left(R_{u}^{\prime} R_{u}^{\prime}\right)
$$

Combining this with the result for the $r^{2}$-coefficient of $-2 \operatorname{Tr}\left(\sigma^{4}\right)$ from (8), we conclude that the terms involving $\operatorname{Tr}\left(R_{u}^{\prime} R_{u}^{\prime}\right)$ in the coefficient of $r^{2}$ in the power series expansion of $\left|R^{S_{r}(p)}\right|_{\exp (r u)}^{2}$ cancel each other, and the only remaining term apart from those which depend solely on $n, C, H, L$ is $\frac{4}{9} \sum_{i=1}^{n} \operatorname{Tr}\left(R_{u} \circ R\left(e_{i},.\right) R_{u} e_{i}\right)$, as claimed. 
Remark 3.3. For the purpose of the proof of the Main Theorem 1.1 in Section 4, which we will perform using the heat invariants $a_{0}\left(S_{r}(p)\right)=\operatorname{vol}\left(S_{r}(p)\right)$ and $a_{2}\left(S_{r}(p)\right)=\frac{1}{360} \int_{S_{r}(p)}\left(5\left(\operatorname{scal}^{S}\right)^{2}-\right.$ $\left.2\left|\operatorname{Ric}^{S}\right|^{2}+2\left|R^{S}\right|^{2}\right) d v o l_{S_{r}(p)}$, we would actually not have needed the exact statement of the previous proposition - which might, however, be interesting in its own right. Rather, we could have restricted our attention to the term $\operatorname{Tr}\left(\sigma^{\prime} \sigma^{\prime}\right)$ in the expression of $\left|\operatorname{Ric}^{S}\right|_{\gamma_{u}(r)}^{2}$ in Lemma 3.1(i), and to the last two terms in the expression of $\left|R^{S}\right|_{\gamma_{u}(r)}^{2}$ in Lemma 3.1(ii). In fact, even without the explicit calculation of the expansion of the other terms, one easily sees that those are determined by the volume function $r \mapsto a_{0}\left(S_{r}(p)\right)=\operatorname{vol}\left(S_{r}(p)\right)$ of the geodesic spheres (which is just the function $v$ multiplied by the volume of the standard unit sphere, see Remark 4.1 below). More precisely, in the spirit of Remark 2.3 we obtain

$$
\begin{aligned}
2 C(\operatorname{Tr}(\sigma))^{2} & =2 C\left(v^{\prime} / v\right)^{2}, \\
(\operatorname{Tr}(\sigma))^{2} \operatorname{Tr}\left(\sigma^{2}\right) & =\left(v^{\prime} / v\right)^{2}\left(-\left(v^{\prime} / v\right)^{\prime}-C\right), \\
2 C \operatorname{Tr}\left(\sigma^{\prime}\right) & =2 C \operatorname{Tr}(\sigma)^{\prime}=2 C\left(v^{\prime} / v\right)^{\prime}, \\
2 \operatorname{Tr}(\sigma) \operatorname{Tr}\left(\sigma \sigma^{\prime}\right) & =2 v^{\prime} / v \cdot \frac{1}{2}\left(\operatorname{Tr}\left(\sigma^{2}\right)\right)^{\prime}=v^{\prime} / v \cdot\left(-\left(v^{\prime} / v\right)^{\prime \prime}\right), \\
2\left(\operatorname{Tr}\left(\sigma^{2}\right)\right)^{2} & =2\left(-\left(v^{\prime} / v\right)^{\prime}-C\right)^{2} .
\end{aligned}
$$

\section{Proof of the Main Theorem}

In this section we will first derive an integrated version of Proposition 3.2. Using this and the heat invariants $a_{0}, a_{1}, a_{2}$ of geodesic spheres in harmonic spaces we will then prove our Main Theorem 1.1. We need the following general remark on mean values.

Remark 4.1. In any harmonic space $M$, the average (or mean value) of a smooth function $f$ on a geodesic sphere $S_{r}(p)$ (with $0<r<i(p)$ ) is the same as the average of $f(\exp (r$.$) ) over$ the unit sphere $S_{1}\left(0_{p}\right)$ in $T_{p} M$. More explicitly: Let $\omega_{n-1}$ denote the volume of the $(n-1)$ dimensional standard sphere. In particular, $\omega_{n-1}$ is the volume of $S_{1}\left(0_{p}\right)$. Recall from Section 2 that $\theta(r)=\theta_{u}(r)$ is independent of $u$ (and even of $p$ ) by harmonicity. We have

$$
\operatorname{vol}\left(S_{r}(p)\right)=r^{n-1} \theta(r) \omega_{n-1}=v(r) \omega_{n-1},
$$

and for any smooth function $f$ on $S_{r}(p)$,

$$
\begin{aligned}
\frac{1}{\operatorname{vol}\left(S_{r}(p)\right)} \int_{S_{r}(p)} f d v o l_{S_{r}(p)} & =\frac{1}{v(r) \omega_{n-1}} \int_{S_{1}\left(0_{p}\right)} f(\exp (r u)) v(r) d u \\
& =\frac{1}{\omega_{n-1}} \int_{S_{1}\left(0_{p}\right)} f(\exp (r u)) d u
\end{aligned}
$$

Now we can give an "integrated" version of Proposition 3.2 ,

Proposition 4.2. Let $M$ be an n-dimensional harmonic space, and let $C, H$, and $L$ be the constants from Proposition 2.1. Let $p \in M$. Then

$$
\begin{aligned}
& \frac{1}{\operatorname{vol}\left(S_{r}(p)\right)} \int_{S_{r}(p)}\left|\operatorname{Ric}^{S_{r}(p)}\right|^{2} d v \operatorname{vol}_{S_{r}(p)}=\alpha_{-4} r^{-4}+\alpha_{-2} r^{-2}+\alpha_{0}+\bar{\alpha}_{2} r^{2}+O\left(r^{3}\right) \text { and } \\
& \frac{1}{\operatorname{vol}\left(S_{r}(p)\right)} \int_{S_{r}(p)}\left|R^{S_{r}(p)}\right|^{2} d v o l_{S_{r}(p)}=\beta_{-4} r^{-4}+\beta_{-2} r^{-2}+\beta_{0}+\bar{\beta}_{2} r^{2}+O\left(r^{3}\right)
\end{aligned}
$$


for $r \downarrow 0$, where the coefficients $\alpha_{i}$ and $\beta_{i}$ for $i \in\{-4,-2,0\}$ are the constants from Proposition 3.2 depending only on $n, C$, and $H$. Moreover,

$$
\begin{aligned}
& \bar{\alpha}_{2}=\tilde{\alpha}_{2}+\frac{3}{16 n(n+2)(n+4)}|\nabla R|_{p}^{2} \text { and } \\
& \bar{\beta}_{2}=\tilde{\beta}_{2}+\frac{1}{8 n(n+2)}|\nabla R|_{p}^{2},
\end{aligned}
$$

where $\tilde{\alpha}_{2}$ and $\tilde{\beta}_{2}$ are constants depending only on $n, C, H$, and $L$.

Proof. For any unit vector $u$ in $T_{p} M$, let $\alpha_{2}(u)$ and $\beta_{2}(u)$ be the coefficients from Proposition 3.2 . Using that proposition and Remark 4.1, we only need to show that

$$
\begin{aligned}
& \bar{\alpha}_{2}:=\frac{1}{\omega_{n-1}} \int_{S_{1}\left(0_{p}\right)} \alpha_{2}(u) d u=\hat{\alpha}_{2}+\frac{1}{\omega_{n-1}} \int_{S_{1}\left(0_{p}\right)} \frac{1}{16} \operatorname{Tr}\left(R_{u}^{\prime} R_{u}^{\prime}\right) d u \text { and } \\
& \bar{\beta}_{2}:=\frac{1}{\omega_{n-1}} \int_{S_{1}\left(0_{p}\right)} \beta_{2}(u) d u=\hat{\beta}_{2}+\frac{1}{\omega_{n-1}} \int_{S_{1}\left(0_{p}\right)} \frac{4}{9} \sum_{i=1}^{n} \operatorname{Tr}\left(R_{u} \circ R\left(e_{i}, .\right) R_{u} e_{i}\right) d u
\end{aligned}
$$

are of the claimed form, where $\hat{\alpha}_{2}, \hat{\beta}_{2}$ are as in Proposition 3.2 . For $\bar{\alpha}_{2}$ this follows immediately (with $\tilde{\alpha}_{2}:=\hat{\alpha}_{2}$ ) from the following formula (see the proof of Theorem 5.7 of [15]; details of the computation can be found on p. 170 of [12]):

$$
\int_{S_{1}\left(0_{p}\right)} \operatorname{Tr}\left(R_{u}^{\prime} R_{u}^{\prime}\right) d u=\frac{3 \omega_{n-1}}{n(n+2)(n+4)}|\nabla R|_{p}^{2}
$$

This confirms the statement concerning $\bar{\alpha}_{2}$.

We now consider $\bar{\beta}_{2}$. Writing $u=\sum_{i=1}^{n} u_{i} e_{i}$ and $R_{i j k \ell}=\left\langle R\left(e_{i}, e_{j}\right) e_{k}, e_{\ell}\right\rangle$ we have

$$
\begin{aligned}
\sum_{i=1}^{n} \operatorname{Tr}\left(R_{u} \circ R\left(e_{i}, .\right) R_{u} e_{i}\right) & =\sum_{i, j, k, \ell=1}^{n}\left\langle R\left(e_{i}, e_{j}\right) e_{k}, e_{\ell}\right\rangle\left\langle R_{u} e_{i}, e_{k}\right\rangle\left\langle R_{u} e_{j}, e_{\ell}\right\rangle \\
& =\sum_{a, b, c, d=1}^{n}\left[\sum_{i, j, k, \ell=1}^{n} R_{i j k \ell} R_{a i b k} R_{c j d \ell}\right] u_{a} u_{b} u_{c} u_{d} .
\end{aligned}
$$

Note that the integral of $u_{a} u_{b} u_{c} u_{d}$ over $S_{1}\left(0_{p}\right)$ is zero whenever $\{a, b, c, d\}$ contains at least three different elements. Abbreviating $A_{a b c d}:=\sum_{i, j, k, \ell=1}^{n} R_{i j k \ell} R_{a i b k} R_{c j d \ell}$ we have, using the Einstein condition and recalling the definition of $\hat{R}$ and $\stackrel{\circ}{R}$ from Section 2 ;

$$
\begin{aligned}
\sum_{a, b=1}^{n} A_{a a b b} & =\sum_{a, b, i, j, k, \ell=1}^{n} R_{i j k \ell} R_{a i a k} R_{b j b \ell}=C^{2} \sum_{i, j, k, \ell=1}^{n} R_{i j k \ell} \delta_{i k} \delta_{j \ell}=C^{2} \sum_{i, j=1}^{n} R_{i j i j}=n C^{3}, \\
\sum_{a, b=1}^{n} A_{a b a b} & =\sum_{a, b, i, j, k, \ell=1}^{n} R_{i j k \ell} R_{a i b k} R_{a j b \ell}=\sum_{a, b, i, j, k, \ell=1}^{n} R_{i j k \ell} R_{a i b k} R_{j a \ell b}=\stackrel{\circ}{R}(p), \\
\sum_{a, b=1}^{n} A_{a b b a} & =\sum_{a, b, i, j, k, \ell=1}^{n} R_{i j k \ell} R_{a i b k} R_{b j a \ell}=\sum_{a, b, i, j, k, \ell=1}^{n} R_{i j k \ell} R_{a i b k} R_{j b \ell a}=\stackrel{\circ}{R}(p)-\frac{1}{4} \hat{R}(p),
\end{aligned}
$$

where for the last equality we have used formula $(2.7)$ (vi) of [17]; see also formula (2.15) of [12]. Let $S^{n-1} \subset R^{n}$ denote the $(n-1)$-dimensional standard sphere. Note that $\int_{S^{n-1}} u_{1}^{2} u_{2}^{2} d u=\frac{\omega_{n-1}}{n(n+2)}$ 
and $\int_{S^{n-1}} u_{1}^{4} d u=\frac{3 \omega_{n-1}}{n(n+2)}$. From the above equations and (17) we thus obtain

$$
\begin{aligned}
\int_{S_{1}\left(0_{p}\right)} \sum_{i=1}^{n} \operatorname{Tr} & \left(R_{u} \circ R\left(e_{i}, .\right) R_{u} e_{i}\right) d u \\
& =\sum_{\substack{a, b \in\{1, \ldots, n\} \\
a \neq b}}\left[A_{a a b b}+A_{a b a b}+A_{a b b a}\right] \int_{S^{n-1}} u_{1}^{2} u_{2}^{2} d u+\sum_{a=1}^{n} A_{a a a a} \int_{S^{n-1}} u_{1}^{4} d u \\
& =\sum_{a, b=1}^{n}\left[A_{a a b b}+A_{a b a b}+A_{a b b a}\right] \frac{\omega_{n-1}}{n(n+2)}=\left(n C^{3}+2 \stackrel{\circ}{R}(p)-\frac{1}{4} \hat{R}(p)\right) \frac{\omega_{n-1}}{n(n+2)}
\end{aligned}
$$

Hence,

(18) $\bar{\beta}_{2}=\hat{\beta}_{2}+\frac{4}{9}\left(n C^{3}+2 \stackrel{\circ}{R}(p)-\frac{1}{4} \hat{R}(p)\right) \frac{1}{n(n+2)}=\hat{\beta}_{2}+\frac{4 C^{3}}{9(n+2)}+\left(\frac{8}{9} \stackrel{\circ}{R}(p)-\frac{1}{9} \hat{R}(p)\right) \frac{1}{n(n+2)}$

Recall from Proposition 2.2(ii), (iii) and equation ([6) that

$$
\begin{aligned}
112 \hat{R}(p)-32 \stackrel{\circ}{R}(p) & =27|\nabla R|_{p}^{2}+\text { some constant depending only on } n, C, H, L \text { and } \\
\hat{R}(p)+4 \stackrel{\circ}{R}(p) & =|\nabla R|_{p}^{2}+\text { some constant depending only on } n, C, H,
\end{aligned}
$$

using which one easily computes that

$$
-\frac{1}{9} \hat{R}(p)+\frac{8}{9} \stackrel{\circ}{R}(p)=\frac{1}{8}|\nabla R|_{p}^{2}+\text { some constant depending only on } n, C, H, L .
$$

Thus we conclude from (18):

$$
\bar{\beta}_{2}=\tilde{\beta}_{2}+\frac{1}{8 n(n+2)}|\nabla R|_{p}^{2}
$$

where $\tilde{\beta}_{2}$ is a constant depending only on $n, C, H, L$.

\section{Proof of the Main Theorem 1.1;}

Let $M_{1}, M_{2}$ be harmonic spaces, $p_{1} \in M_{1}, p_{2} \in M_{2}$, and assume there exists $\varepsilon$ in the interval $\left(0, \min \left\{i\left(p_{1}\right), i\left(p_{2}\right)\right\}\right)$ such that for each $0<r<\varepsilon$ the geodesic spheres $S_{r}\left(p_{1}\right)$ and $S_{r}\left(p_{2}\right)$ are isospectral. Then $\operatorname{dim} M_{1}=: n=\operatorname{dim} M_{2}$, and the heat invariants of the geodesic spheres coincide:

$$
a_{k}\left(S_{r}\left(p_{1}\right)\right)=a_{k}\left(S_{r}\left(p_{2}\right)\right)
$$

for each $r \in(0, \varepsilon)$ and all $k \in \mathbb{N}_{0}$. We want to deduce that $|\nabla R|_{p_{1}}^{2}=|\nabla R|_{p_{2}}^{2}$. Actually this will follow using just $a_{0}$ and $a_{2}$.

Reformulating the problem, let $M$ be an $n$-dimensional harmonic space and $p \in M$. We want to show that for any $\varepsilon \in(0, i(p))$, the two functions

$$
\varphi_{k}:(0, \varepsilon) \ni r \mapsto a_{k}\left(S_{r}(p)\right) \in \mathbb{R}
$$

with $k \in\{0,2\}$ together determine the value of $|\nabla R|_{p}^{2}$. By Remark 2.3(i), the function

$$
\varphi_{0}: r \mapsto a_{0}\left(S_{r}(p)\right)=\operatorname{vol}\left(S_{r}(p)\right)=v(r) \omega_{n-1}
$$

determines the constants $C, H, L$ associated with $M$ (see Section 2). Recall that the scalar curvature scal $^{S}=: \mathrm{scal}^{S_{r}}$ of $S_{r}(p)$ is constant on the manifold $S_{r}(p)$, and that the function $v:(0, \varepsilon) \rightarrow \mathbb{R}$ determines, by Remark 2.3(ii), the function $(0, \varepsilon) \ni r \mapsto \mathrm{scal}^{S_{r}} \in \mathbb{R}$. In particular, the function 

$\varphi_{0}=v \omega_{n-1}$ also determines the function $(0, \varepsilon) \ni r \mapsto \int_{S_{r}(p)}\left(\operatorname{scal}^{S}\right)^{2} d v o l_{S_{r}(p)}=\varphi_{0}(r) \cdot\left(\operatorname{scal}^{S_{r}}\right)^{2} \in \mathbb{R}$. By

$$
\varphi_{2}(r)=a_{2}\left(S_{r}(p)\right)=\frac{1}{360} \int_{S_{r}(p)}\left(5\left(\mathrm{scal}^{S}\right)^{2}-2\left|\operatorname{Ric}^{S}\right|^{2}+2\left|R^{S}\right|^{2}\right) d v o l_{S_{r}(p)}
$$

it follows that the functions $\varphi_{0}$ and $\varphi_{2}$ together determine the function

$$
(0, \varepsilon) \ni r \mapsto \frac{1}{\operatorname{vol}\left(S_{r}(p)\right)} \int_{S_{r}(p)}\left(\left|R^{S}\right|^{2}-\left|\operatorname{Ric}^{S}\right|^{2}\right) d v o l_{S_{r}(p)} \in \mathbb{R} .
$$

By Proposition 4.2, the $r^{2}$-coefficient in the power series expansion of this function is the sum of the term

$$
\left(\frac{1}{8 n(n+2)}-\frac{3}{16 n(n+2)(n+4)}\right)|\nabla R|_{p}^{2}=\frac{2 n+5}{16 n(n+2)(n+4)}|\nabla R|_{p}^{2}
$$

and $\tilde{\beta}_{2}-\tilde{\alpha}_{2}$. Recall that the latter is a constant depending only on $n, C, H, L$, and is thus determined by $\varphi_{0}$. We conclude that the functions $\varphi_{0}$ and $\varphi_{2}$ together determine $|\nabla R|_{p}^{2}$, as claimed.

\section{Geodesic Balls}

In this section we will prove the following version of the Main Theorem 1.1 for geodesic balls:

Theorem 5.1. Let $M_{1}$ and $M_{2}$ be harmonic spaces, and let $p_{1} \in M_{1}, p_{2} \in M_{2}$. If there exists $\varepsilon>0$ such that for each $r \in(0, \varepsilon)$ the geodesic balls $B_{r}\left(p_{1}\right)$ and $B_{r}\left(p_{2}\right)$ are Dirichlet isospectral, then $|\nabla R|_{p_{1}}^{2}=|\nabla R|_{p_{2}}^{2}$. The same holds if the assumption of Dirichlet isospectrality is replaced by the assumption of Neumann isospectrality.

This theorem implies the corresponding analog of our Main Corollary 1.2.

Corollary 5.2. Let $M_{1}$ and $M_{2}$ be harmonic spaces. Assume that the Dirichlet isospectrality hypothesis of Theorem 5.1 is satisfied for each pair of points $p_{1} \in M_{1}, p_{2} \in M_{2}$. Then $M_{1}$ is locally symmetric if and only if $M_{2}$ is locally symmetric. The same holds if the assumption of Dirichlet isospectrality is replaced by the assumption of Neumann isospectrality.

For the proof of Theorem 5.1 we will use the heat invariants for manifolds with boundary. Let $M$ be an $n$-dimensional Riemannian manifold, and let $B \subset M$ be a compact domain with smooth boundary. If $\Delta$ denotes the Laplace operator on $B$ with Dirichlet boundary conditions then there is an asymptotic expansion

$$
\operatorname{Tr}(\exp (-t \Delta)) \sim(4 \pi t)^{-n / 2} \sum_{k} a_{k}^{D}(B) t^{k}
$$

for $t \downarrow 0$, where $k=0,0.5,1,1.5, \ldots$ ranges over the nonnegative half integers (see [3]). For the Laplace operator on $B$ with Neumann boundary conditions the analog of this formula holds with certain coefficients $a_{k}^{N}(B)$. The coefficients $a_{k}^{D}(B)$ (resp. $a_{k}^{N}(B)$ ) are given by certain curvature integrals over $B$ and $\partial B$. One has $a_{0}^{D}(B)=a_{0}^{N}(B)=\operatorname{vol}(B)$ and $a_{0.5}^{D}(B)=-a_{0.5}^{N}(B)=-\frac{\sqrt{\pi}}{2} \operatorname{vol}(\partial B)$ (see [3]). In the proof of Theorem [5.1 we will use the explicit formulas for $a_{2}^{D}(B)$ and $a_{2}^{N}(B)$ from [3]. Let $\nu$ denote the outward pointing unit vector field on the boundary $\partial B$ of $B$, and let $\sigma=\nabla \nu$ be the associated shape operator. Let scal, Ric, $R$ always refer to the usual objects on $M$ 
(not to the ones associated with the induced metric on $\partial B$ ). Then

$$
\begin{aligned}
& a_{2}^{D}(B)=\frac{1}{360}\left[\int_{B}\left(-12 \Delta(\mathrm{scal})+5 \mathrm{scal}^{2}-2|\mathrm{Ric}|^{2}+2|R|^{2}\right) d v o l_{B}\right. \\
& +\int_{\partial B}\left(18 \nu(\mathrm{scal})+20 \mathrm{scal} \cdot \operatorname{Tr}(\sigma)-4 \operatorname{Tr}\left(R_{\nu}\right) \operatorname{Tr}(\sigma)+12 \operatorname{Tr}\left(R_{\nu} \circ \sigma\right)\right. \\
& \left.\left.-4 \operatorname{Tr}\left(\left(\operatorname{Ric}-R_{\nu}\right) \circ \sigma\right)+\frac{40}{21}(\operatorname{Tr}(\sigma))^{3}-\frac{88}{7} \operatorname{Tr}(\sigma) \operatorname{Tr}\left(\sigma^{2}\right)+\frac{320}{21} \operatorname{Tr}\left(\sigma^{3}\right)\right) d v o l_{\partial B}\right], \\
& a_{2}^{N}(B)=\frac{1}{360}\left[\int_{B}\left(-12 \Delta(\mathrm{scal})+5 \mathrm{scal}^{2}-\left.2\left|\mathrm{Ric}^{2}+2\right| R\right|^{2}\right) d v o l_{B}\right. \\
& +\int_{\partial B}\left(-42 \nu(\mathrm{scal})+20 \mathrm{scal} \cdot \operatorname{Tr}(\sigma)-4 \operatorname{Tr}\left(R_{\nu}\right) \operatorname{Tr}(\sigma)+12 \operatorname{Tr}\left(R_{\nu} \circ \sigma\right)\right. \\
& \left.\left.-4 \operatorname{Tr}\left(\left(\operatorname{Ric}-R_{\nu}\right) \circ \sigma\right)+\frac{40}{3}(\operatorname{Tr}(\sigma))^{3}+8 \operatorname{Tr}(\sigma) \operatorname{Tr}\left(\sigma^{2}\right)+\frac{32}{3} \operatorname{Tr}\left(\sigma^{3}\right)\right) d \text { vol }_{\partial B}\right] .
\end{aligned}
$$

If $M$ is harmonic then, by the results of Section 2 , the previous formulas simplify to

$$
\begin{aligned}
a_{2}^{D}(B)= & \frac{1}{360}\left[\operatorname{vol}(B) \cdot\left(5(n C)^{2}-2 n C^{2}+\frac{4}{3} n\left((n+2) H-C^{2}\right)\right)\right. \\
+ & \int_{\partial B}\left(20 n C \operatorname{Tr}(\sigma)-8 C \operatorname{Tr}(\sigma)+16 \operatorname{Tr}\left(R_{\nu} \circ \sigma\right)\right. \\
& \left.\left.+\frac{40}{21}(\operatorname{Tr}(\sigma))^{3}-\frac{88}{7} \operatorname{Tr}(\sigma) \operatorname{Tr}\left(\sigma^{2}\right)+\frac{320}{21} \operatorname{Tr}\left(\sigma^{3}\right)\right) d v o l_{\partial B}\right] \\
a_{2}^{N}(B)= & \frac{1}{360}\left[\operatorname{vol}(B) \cdot\left(5(n C)^{2}-2 n C^{2}+\frac{4}{3} n\left((n+2) H-C^{2}\right)\right)\right. \\
+ & \int_{\partial B}\left(20 n C \operatorname{Tr}(\sigma)-8 C \operatorname{Tr}(\sigma)+16 \operatorname{Tr}\left(R_{\nu} \circ \sigma\right)\right. \\
& \left.\left.\quad+\frac{40}{3}(\operatorname{Tr}(\sigma))^{3}+8 \operatorname{Tr}(\sigma) \operatorname{Tr}\left(\sigma^{2}\right)+\frac{32}{3} \operatorname{Tr}\left(\sigma^{3}\right)\right) d v o l_{\partial B}\right]
\end{aligned}
$$

In the proof of Theorem [5.1 we will follow a similar strategy as in the proof of Theorem 1.1. To this end, we need some preliminary results in the special case that $B=B_{r}(p)$ with $r \in(0, i(p))$ and $M$ is harmonic. We remark - without going into details this time - that one can compute in this case, using equation (44), Proposition 2.1, and the Taylor series expansion

$$
P_{\gamma_{u}}^{r, 0} \circ\left(R_{\nu}\right)_{\gamma_{u}(r)} \circ P_{\gamma_{u}}^{0, r}=\sum_{k=0}^{\infty} \frac{r^{k}}{k !} R_{u}^{(k)},
$$

that the $r^{3}$-coefficient in the power series expansion of $\operatorname{Tr}\left(R_{\nu} \circ \sigma\right)$ equals $-\frac{1}{1440} L+\frac{1}{96} \operatorname{Tr}\left(R_{u}^{\prime} R_{u}^{\prime}\right)$, and that the $r^{3}$-coefficient in the power series expansion of $\operatorname{Tr}\left(\sigma^{3}\right)$ equals $\frac{1}{30240} L-\frac{1}{96} \operatorname{Tr}\left(R_{u}^{\prime} R_{u}^{\prime}\right)$. (That the contributions of $\operatorname{Tr}\left(R_{u}^{\prime} R_{u}^{\prime}\right)$ in these terms are negatives of each other can also be checked as follows: Using (3) twice, we have $\operatorname{Tr}\left(R_{\nu} \circ \sigma\right)+\operatorname{Tr}\left(\sigma^{3}\right)=-\operatorname{Tr}\left(\sigma^{\prime} \sigma\right)=-\frac{1}{2} \operatorname{Tr}\left(\sigma^{2}\right)^{\prime}=\frac{1}{2} \operatorname{Tr}\left(R_{\nu}+\sigma^{\prime}\right)^{\prime}=$ $\frac{1}{2} \operatorname{Tr}(\sigma)^{\prime \prime}$ whose $r^{3}$-coefficient indeed depends only on $L$ by (5).) Using (16), we conclude that the $r^{3}$-coefficient in the power series expansion of $r \mapsto \frac{1}{v o l\left(S_{r}(p)\right)} \int_{S_{r}(p)} \operatorname{Tr}\left(R_{\nu} \circ \sigma\right) d v o l_{S_{r}(p)}$ is

$$
-\frac{1}{1440} L+\frac{1}{32 n(n+2)(n+4)}|\nabla R|_{p}^{2}
$$


Similarly, the $r^{3}$-coefficient in the power series expansion of $r \mapsto \frac{1}{v o l\left(S_{r}(p)\right)} \int_{S_{r}(p)} \operatorname{Tr}\left(\sigma^{3}\right) d v o l_{S_{r}(p)}$ is

$$
\frac{1}{30240} L-\frac{1}{32 n(n+2)(n+4)}|\nabla R|_{p}^{2} .
$$

\section{Proof of Theorem 5.1;}

Let $M_{1}, M_{2}$ be harmonic spaces, $p_{1} \in M_{1}, p_{2} \in M_{2}$, and assume there exists $\varepsilon$ in the interval $\left(0, \min \left\{i\left(p_{1}\right), i\left(p_{2}\right)\right\}\right)$ such that for each $0<r<\varepsilon$ the geodesic spheres $B_{r}\left(p_{1}\right)$ and $B_{r}\left(p_{2}\right)$ are Dirichlet isospectral (resp. Neumann isospectral). Then $\operatorname{dim} M_{1}=: n=\operatorname{dim} M_{2}$, and the heat invariants of the geodesic spheres coincide:

$$
a_{k}^{D}\left(B_{r}\left(p_{1}\right)\right)=a_{k}^{D}\left(B_{r}\left(p_{2}\right)\right), \quad \text { resp. } a_{k}^{N}\left(B_{r}\left(p_{1}\right)\right)=a_{k}^{N}\left(B_{r}\left(p_{2}\right)\right)
$$

for each $r \in(0, \varepsilon)$ and all $k \in \mathbb{N}_{0}$. We want to deduce that $|\nabla R|_{p_{1}}^{2}=|\nabla R|_{p_{2}}^{2}$. Actually this will follow using just $a_{0}$ and $a_{2}$. (We remark without proof here that, viewed as functions of $r$, the heat coefficients $a_{0.5}, a_{1}$, and $a_{1.5}$ do actually not contain more information than $a_{0}$ in our situation.)

We first consider the case of Dirichlet conditions. Similarly as in the proof of Theorem 1.1, we reformulate the problem as follows: Let $M$ be an $n$-dimensional harmonic space and $p \in M$. We want to show that for any $\varepsilon \in(0, i(p))$, the two functions

$$
\psi_{k}^{D}:(0, \varepsilon) \ni r \mapsto a_{k}^{D}\left(B_{r}(p)\right) \in \mathbb{R}
$$

with $k \in\{0,2\}$ together determine the value of $|\nabla R|_{p}^{2}$. Note that the function

$$
\psi_{0}^{D}: r \mapsto a_{0}^{D}\left(B_{r}(p)\right)=\operatorname{vol}\left(B_{r}(p)\right)
$$

determines its own derivative which is just

$$
r \mapsto \operatorname{vol}\left(S_{r}(p)\right)=v(r) \omega_{n-1}
$$

(see the previous section). By Remark 2.3(i), we conclude that $\psi_{0}^{D}$ again determines the constants $C, H, L$ associated with $M$. Moreover, the function $v:(0, \varepsilon) \rightarrow \mathbb{R}$ determines the radial functions $\operatorname{Tr}(\sigma)=v^{\prime} / v$ and $\operatorname{Tr}\left(\sigma^{2}\right)=-\left(v^{\prime} / v\right)^{\prime}-C$ (compare Remark 3.3). By (19) it now follows that $\psi_{0}^{D}$ and $\psi_{2}^{D}$ together determine the function

$$
(0, \varepsilon) \ni r \mapsto \frac{1}{\operatorname{vol}\left(S_{r}(p)\right)} \int_{S_{r}(p)}\left(16 \operatorname{Tr}\left(R_{\nu} \circ \sigma\right)+\frac{320}{21} \operatorname{Tr}\left(\sigma^{3}\right)\right) d v \operatorname{vol}_{S_{r}(p)} \in \mathbb{R} .
$$

Recalling (21) and (22), we see that the $r^{3}$-coefficient in the power series expansion of the latter function is the sum of

$$
\frac{1}{32 n(n+2)(n+4)}\left(16-\frac{320}{21}\right)|\nabla R|_{p}^{2}=\frac{1}{42 n(n+2)(n+4)}|\nabla R|_{p}^{2}
$$

and a term depending only on $L$. Since $L$ is determined by $\psi_{0}^{D}$, we conclude that $\psi_{0}^{D}$ and $\psi_{2}^{D}$ together determine $|\nabla R|_{p}^{2}$, as claimed.

In the Neumann case, letting $\psi_{k}^{N}(r):=a_{k}^{N}\left(B_{r}(p)\right)$, we again have $\psi_{0}^{N}(r)=\operatorname{vol}\left(B_{r}(p)\right)=\psi_{0}^{D}(r)$. Proceeding exactly as in the Dirichlet case, using (20) this time, we see that $\psi_{0}^{N}$ and $\psi_{2}^{N}$ together determine the sum of

$$
\frac{1}{32 n(n+2)(n+4)}\left(16-\frac{32}{3}\right)|\nabla R|_{p}^{2}=\frac{1}{6 n(n+2)(n+4)}|\nabla R|_{p}^{2}
$$

and a term depending only on $L$. Hence they determine $|\nabla R|_{p}^{2}$, as claimed. 


\section{REFERENCES}

[1] J. Berndt, F. Tricerri, L. Vanhecke, Generalized Heisenberg groups and Damek-Ricci harmonic spaces, Lecture Notes in Mathematics 1598, Springer-Verlag, Berlin/Heidelberg/New York, 1995.

[2] A.L. Besse, Manifolds all of whose geodesics are closed, Ergebnisse der Mathematik und ihrer Grenzgebiete 93, Springer-Verlag, Berlin/New York, 1978.

[3] T. Branson, P.B. Gilkey, The asymptotics of the Laplacian on a manifold with boundary, Comm. Partial Differential Equations 15 (1990), no. 2, 245-272.

[4] B.-Y. Chen, L. Vanhecke, Differential geometry of geodesic spheres, J. Reine Angew. Math. 325 (1981), $28-67$.

[5] E.T. Copson, H.S. Ruse, Harmonic Riemannian spaces, Proc. Roy. Soc. Edinburgh 60 (1940), $117-133$.

[6] E. Damek, F. Ricci, A class of nonsymmetric harmonic Riemannian spaces, Bull. Amer. Math. Soc. (N.S.) 27 (1992), no. 1, 139-142.

[7] D. DeTurck, J. Kazdan, Some regularity theorems in Riemannian geometry, Ann. scient. Éc. Norm. Sup. (4) 14 (1981), 249-260.

[8] H. Fürstenau, Über Isospektralität von topologischen Bällen, Diploma thesis, Universität Bonn, 2006.

[9] P. Gilkey, Invariance Theory, the Heat Equation, and the Atiyah-Singer Index Theorem, Mathematics Lecture Series 11, Publish or Perish, Wilmington, Del., 1984.

[10] C. Gordon, Survey of isospectral manifolds, Handbook of differential geometry, Vol. I, 747-778, North-Holland, Amsterdam, 2000.

[11] C. Gordon, Isospectral deformations of metrics on spheres, Invent. Math. 145 (2001), 317-331.

[12] A. Gray, L. Vanhecke, Riemannian geometry as determined by the volumes of small geodesic balls, Acta Math. 142 (1979), no. 3-4, 157-198.

[13] A. Lichnerowicz, Sur les espaces Riemanniens complètement harmoniques, Bull. Soc. Math. France 72 (1944), $146-168$.

[14] A. Lichnerowicz, Géométrie des groupes de transformations, Dunod, Paris, 1958.

[15] L. Nicolodi, L. Vanhecke, The geometry of k-harmonic manifolds, Adv. Geom. 6 (2006), no. 1, 53-70.

[16] H.S. Ruse, A.G. Walker, T.J. Willmore, Harmonic Spaces, Consiglio Nazionale delle Ricerche, Monografie Matematice 8, Rome, 1961.

[17] T. Sakai, On eigen-values of Laplacian and curvature of Riemannian manifolds, Tôhoku Math. J. (2) 23 (1971), 589-603.

[18] Z.I. Szabó, The Lichnerowicz conjecture on harmonic manifolds, J. Differential Geom. 31 (1990), no. 1, 1-28.

[19] Z.I. Szabó, Locally non-isometric yet super isospectral spaces, Geom. Funct. Anal. 9 (1999), no. 1, $185-214$.

[20] Z.I. Szabó, Isospectral pairs of metrics on balls, spheres, and other manifolds with different local geometries, Ann. of Math. (2) 154 (2001), no. 2, 437-475.

[21] Z.I. Szabó, A cornucopia of isospectral pairs of metrics on spheres with different local geometries, Ann. of Math. (2) 161 (2005), no. 1, 343-395.

[22] Y. Watanabe, On the characteristic function of harmonic Kählerian spaces, Tôhoku Math. J. (2) 27 (1975), $13-24$.

Departamento de Matemáticas, Universidad de Extremadura, 06071 Badajoz, Spain

E-mail address: ariasmarco@unex.es

Institut für Mathematik, Humboldt-Universität Zu Berlin, D-10099 Berlin, Germany

E-mail address: schueth@math.hu-berlin.de 